\author{
Ефанов М.Г. • Алиханов Р.Б. ${ }^{1}$ • Цвиркун В.В.' • Казаков И.В.' • Ким П.П. • Ванькович А.Н.' • \\ Грендаль К.Д.' $\cdot$ Заманов Э.Н.2
}

Ефанов Михаил Германович - д-р мед. наук, руководитель отдела гепатопанкреатобилиарной хирургии' $\triangle 111123$, г. Москва, шоссе

Энтузиастов, 86, Российская Федерация. Тел.: +7 (916) 1058830.

E-mail:m.efanov@mknc.ru

Алиханов Руслан Богданович - канд. мед. наук, заведующий отделением гепатопанкреатобилиарной хирургии'

Цвиркун Виктор Викторович - д-р мед. наук, профессор, гл. науч. сотр. ${ }^{1}$

Казаков Иван Вячеславович - канд. мед. наук, ст. науч. сотр. отделения гепатопанкреатобилиарной хирургии'

Ким Павел Петрович - науч. сотр. отделения гепатопанкреатобилиарной хирургии ${ }^{1}$

Ванькович Андрей Николаевич канд. мед. наук, науч. сотр. отделения гепатопанкреатобилиарной хирургии' Грендаль Константин Дмитриевич клинический аспирант кафедры факультетской хирургии № 2 лечебного факультета ${ }^{2}$

Заманов Эхтибар Низамиевич клинический аспирант кафедры факультетской хирургии № 2 лечебного факультета ${ }^{2}$

1 ГБУЗ г. Москвы «Московский клинический научно-практический центр имени А.С. Логинова Департамента здравоохранения г. Москвы»; 111123 , г. Москва, шоссе Энтузиастов, 86, Российская Федерация

${ }^{2}$ ФГБОУ ВО «Московский государственный медико-стоматологический университет имени А.И. Евдокимова» Минздрава России; 127473, г. Москва, ул. Делегатская, 20-1, Российская Федерация
Актуальность. До настоящего времени вопрос о безопасности лапароскопических резекций печени, в том числе сложных сегментов, недостаточно изучен. Цель - оценить у пациентов с метастазами колоректального рака ближайшие результаты минимально инвазивных и открытых резекций печени, выполненных в российском центре хирургической гепатологии, специализированном на внедрении минимально инвазивных технологий. Материал и методы. Проведено ретроспективное наблюдательное исследование по типу «случай - контроль». В исследование включены пациенты, оперированные в одном центре с октября 2013 по февраль 2018 г. по поводу изолированных метастазов колоректального рака в печень. Результаты. На декабрь 2017 г. в центре выполнено более 500 резекций печени, из них минимально инвазивных - 226. Резекции печени (открытые и минимально инвазивные) по поводу метастазов колоректального рака проведены 102 пациентам. Из 83 пациентов, включенных в исследование, минимально инвазивные резекции печени выполнены у 51 (61\%) больного, из них 7 робот-ассистированных. Группы открытых и минимально инвазивных резекций не различались между собой по полу, возрасту, оценке физического состояния по шкале ASA, по локализации первичной опухоли и стадии онкологического заболевания на момент первичного вмешательства. Отсутствовали межгрупповые различия по факторам, определяющим степень распространенности опухоли и, соответственно, сложность резекции: индексу сложности лапароскопической резекции печени, частоте анатомических резекций, резекций сложных сегментов, контакту с сосудами, размеру и числу метастазов, множественному характеру поражения печени и билобарным метастазам.
При сравнении непосредственных результатов не выявлено различий по частоте отступа плоскости резекции от края опухоли более 2 мм, частоте применения приема Прингла, длительности операции, частоте переливания компонентов крови, частоте тяжелых осложнений (более II степени по Clavien - Dindo), длительности нахождения в отделении реанимации и интенсивной терапии. Минимально инвазивные резекции сопровождались статистически значимо меньшей кровопотерей по сравнению с открытыми: 308 (0-3300) мл и 583 (50-3000) мл ( $p=0,012)$ соответственно, а также более коротким стационарным лечением: 9 (4-29) и 10 (4-29) дней $(p<0,001)$ соответственно. Заключение. В условиях специализированного центра хирургической гепатологии уровень технического исполнения резекции печени может быть повышен до сопоставимого со сложными открытыми операциями без изменения частоты и характера осложнений, но с улучшением непосредственных результатов.

Ключевые слова: минимально инвазивная резекция печени, лапароскопическая резекция печени, метастазы колоректального рака

Для цитирования: Ефанов МГ, Алиханов РБ, Цвиркун ВВ, Казаков ИВ, Ким ПП, Ванькович АН, Грендаль КД, Заманов ЭН. Ближайшие результаты минимально инвазивных и открытых резекций печени по поводу метастазов колоректального рака. Опыт специализированного центра. Альманах клинической медицины. 2018;46(6):584-91. doi: 10.18786/2072-0505-2018-46-6-584-591.

Поступила 09.06.2018;

принята к публикации 06.08.2018 
И стория интенсивного накопления опыта применения минимально инвазивных технологий в абдоминальной хирургии насчитывает более четверти века. Сегодня лапароскопический доступ занимает прочные позиции в различных отраслях абдоминальной хирургии. Стартовав практически одновременно в начале 90-х гг. прошлого столетия, несколько направлений лапароскопической хирургии получили в дальнейшем неодинаковое развитие [1]. Лапароскопическая резекционная хирургия печени, развиваясь более медленно, в определенной степени повторила историю становления классической открытой хирургии печени, получившей второй импульс спустя более чем полвека после выполнения первых успешных резекций печени. В последние годы происходит бурное развитие лапароскопической хирургии печени, аналогичное этапу, который пережила лапароскопическая хирургия полых органов более десяти лет назад. Очевидно, что некоторый временной «сдвиг» в освоении новых методов как классической, так и минимально инвазивной резекционной хирургии печени, наблюдаемый по отношению к желудочной и колоректальной хирургии, обусловлен сложностью хирургической анатомии печени, необходимостью более длительного накопления опыта, адаптации возможностей лапароскопического доступа к принципам хирургии печени. В связи с этим возросло внимание исследователей к вопросам безопасности и радикальности минимально инвазивных резекций печени, стали активно изучаться возможности такого доступа при выполнении сложных операций на печени, анализироваться кривые обучения различным вариантам минимально инвазивного доступа в резекционной хирургии печени. Многие исследования носят ретроспективный характер, проспективные рандомизированные исследования единичны $[2,3]$. Тем не менее растет число метааналитических обзоров [4]. Быстрому накоплению опыта препятствует относительно небольшая численность центров, располагающих регулярной практикой лапароскопических резекций на печени.

Наибольший интерес представляет эффективность и онкологическая радикальность лапароскопических резекций при злокачественных опухолях печени. Немаловажное условие проведения полноценного сравнения технологий преодоление первоначального этапа освоения минимально инвазивных резекций печени, что возможно в условиях крупного специализированного центра.
Целью настоящего исследования была оценка у пациентов с метастазами колоректального рака ближайших результатов минимально инвазивных и открытых резекций печени, выполненных в отечественном центре хирургической гепатологии, специализированном на внедрении минимально инвазивных технологий.

\section{Материал и методы}

В исследование включены пациенты, оперированные в одном центре (ГБУЗ г. Москвы «Московский клинический научно-практический центр имени А.С. Логинова Департамента здравоохранения г. Москвы») с октября 2013 по февраль 2018 г. по поводу метастазов колоректального рака в печень. Все пациенты оперированы двумя хирургами или с их непосредственным участием. На момент проведения исследования общее число выполненных в центре минимально инвазивных резекций составило более 200, в связи с чем сравнение с открытыми резекциями проводилось по истечении первоначального периода кривой обучения, составившей в среднем около 50 операций (вместе с тем часть пациентов с метастазами колоректального рака были оперированы в том числе в период становления методики минимально инвазивных резекций печени в центре). Сравнение проведено по изначальным параметрам, характеризующим сопоставимость групп в отношении переносимости операции, а также по факторам, отражающим степень местного распространения метастатического поражения печени и сложность выполнения резекции печени. Для оценки сложности резекции и связанных с ней рисков использован интегральный показатель, учитывающий несколько параметров, определяющих трудность выполнения резекции, - так называемый индекс сложности лапароскопической резекции печени (ИСЛРП), разработанный D. Ban и соавт. в 2014 г. [5]. ИСЛРП учитывает размер опухоли, топографию опухоли в печени (пораженные сегменты), близость к магистральным сосудам, характер резекции (анатомический или атипичный) и сохранность функции печени. По нескольким из этих параметров сравнение проведено отдельно. В итоге дана сравнительная оценка непосредственных результатов операций: величины отступа от края опухоли, длительности операции, объема кровопотери, частоты тяжелых осложнений по шкале Clavien - Dindo (более II степени), длительности послеоперационного стационарного лечения и других параметров.

Статистическая обработка данных проведена с использованием пакета программы Statistica 12. 
Непрерывные переменные представлены в виде средних величин с интервалами, включающими минимальные и максимальные значения для количественных переменных. Сравнение непрерывных переменных проводилось с использованием U-теста Манна - Уитни. Двузначные категориальные переменные сравнивались при помощи точного критерия Фишера. Статистически значимым считали значение $p$ менее 0,05.

\section{Результаты}

На декабрь 2017 г. в центре выполнено более 500 резекций печени, из них минимально инвазивных - 226. Резекции печени (открытые и минимально инвазивные) по поводу метастазов колоректального рака проведены 102 пациентам. Из анализа исключены пациенты с преобладающим внепеченочным метастатическим распространением опухоли, которым симультанно осуществлены операции резекции печени и удаления внепеченочных очагов опухоли.

Из 83 пациентов, включенных в исследование, минимально инвазивные резекции печени выполнены у 51 (61\%) больного, из них 7 робот-ассистированных, которые применялись на ранних этапах освоения минимально инвазивного доступа, преимущественно при поражении задне-верхних сегментов. Группы открытых

Таблица 1. Основные демографические данные включенных в исследование пациентов и в группах

\begin{tabular}{|c|c|c|c|c|}
\hline \multirow[t]{2}{*}{ Параметр } & \multirow[t]{2}{*}{ Все пациенты } & \multicolumn{2}{|c|}{ Группа исследования } & \multirow[t]{2}{*}{$p$} \\
\hline & & $\begin{array}{c}\text { минимально } \\
\text { инвазивные } \\
\text { резекции }\end{array}$ & $\begin{array}{l}\text { открытые } \\
\text { резекции }\end{array}$ & \\
\hline Пол, женщины / мужчины & $51 / 32$ & $31 / 20$ & $20 / 12$ & 0,876 \\
\hline Средний возраст, годы (min - max) & $60(39-84)$ & $59(41-84)$ & $62(39-79)$ & 0,084 \\
\hline ASA, $1-2 / 3-4$ & $31 / 52$ & $23 / 28$ & $8 / 24$ & 0,065 \\
\hline Опухоль ободочной / прямой кишки & $58 / 25$ & $35 / 16$ & $23 / 9$ & 0,754 \\
\hline Стадия первичной опухоли, 1-2/3-4 & $23 / 60$ & $14 / 37$ & $9 / 23$ & 0,948 \\
\hline
\end{tabular}

ASA - оценка физического статуса пациентов по шкале Американской ассоциации анестезиологов (American Society of Anaesthesiologists)

Таблица 2. Исходные предоперационные параметры метастатического поражения печени в группах

\begin{tabular}{|c|c|c|c|c|}
\hline \multirow[t]{2}{*}{ Параметр } & \multirow[t]{2}{*}{ Все пациенты } & \multicolumn{2}{|l|}{ Группа исследования } & \multirow[t]{2}{*}{$p$} \\
\hline & & $\begin{array}{l}\text { минимально } \\
\text { инвазивные резекции }\end{array}$ & $\begin{array}{l}\text { открытые } \\
\text { резекции }\end{array}$ & \\
\hline ИСЛРП, баллы, М (min - max) & $6,36(2,47-11,08)$ & $6,41(2,47-11,08)$ & $6,27(2,638-10,71)$ & 0,822 \\
\hline Анатомические резекции, n (\%) & $44(53)$ & $28(55)$ & $16(50)$ & 0,809 \\
\hline $\begin{array}{l}\text { Резекции труднодоступных сегментов, } \\
\text { n (\%) }\end{array}$ & $55(66)$ & $35(68)$ & $20(63)$ & 0,795 \\
\hline Контакт метастазов с сосудами, n (\%) & $20(24)$ & $11(22)$ & $9(28)$ & 0,597 \\
\hline Размер метастазов, мм, M (min - max) & $47(8-120)$ & $43(8-96)$ & $55(10-120)$ & 0,121 \\
\hline Число метастазов, n, M (min - max) & $2(1-9)$ & $3(1-9)$ & $2(1-9)$ & 0,940 \\
\hline Множественные метастазы, n (\%) & $31(37)$ & $20(39)$ & $11(34)$ & 0,764 \\
\hline Билобарные метастазы, n (\%) & $38(46)$ & $23(45)$ & $15(47)$ & 0,923 \\
\hline
\end{tabular}

ИСЛРП - индекс сложности лапароскопической резекции печени; труднодоступные сегменты - I, IVa, VII, VIII; контакт метастазов с сосудами тесное прилежание опухоли к магистральным сосудам печени с инвазией / без инвазии; множественные метастазы - 3 и более очагов 
и минимально инвазивных резекций не различались между собой по полу, возрасту, оценке физического состояния по шкале ASA (American Society of Anaesthesiologists), по локализации первичной опухоли и стадии онкологического заболевания на момент первичного вмешательства (табл. 1). Имела место тенденция к более старшему возрасту и большему числу баллов по ASA в группе больных, перенесших открытые вмешательства. Отсутствовали межгрупповые различия между факторами, определяющими степень распространенности опухоли и, соответственно, сложность резекции (табл. 2).

Сравнение непосредственных результатов открытых и минимально инвазивных резекций печени приведено в табл. 3. Из ее данных видно, что минимально инвазивные резекции сопровождались статистически значимо меньшим объемом интраоперационной кровопотери и меньшей длительностью послеоперационного стационарного лечения. Онкологическая оценка ближайших результатов (величина отступа края резекции от поверхности опухоли) показала идентичные результаты для обоих вариантов доступа. Частота тяжелых осложнений (более II степени по Clavien - Dindo), представленных в основном IIIа степенью, статистически значимо не различалась. Детальная характеристика осложнений дана в табл. 4 и 5. При общей тенденции к меньшей частоте осложнений после минимально инвазивных резекций в этой группе чаще наблюдали биломы.

В каждой группе зарегистрировано по одному летальному исходу вследствие острой печеночной недостаточности, обусловленной нарушением кровоснабжения печени.

\section{Обсуждение}

Минимально инвазивная резекция печени в силу специфики применения инструментария требует определенного периода овладения технологий вмешательства. Согласно публикациям ряда зарубежных авторов, число лапароскопических резекций, необходимых для прохождения начального периода обучения, представляющего собой время, затраченное на освоение технически несложных резекций, составляет порядка 40-50 операций $[6,7]$. В дальнейшем следует период расширения показаний, когда результаты операций несколько ухудшаются в силу повышения технической сложности резекции печени. Число операций, необходимых для преодоления этого периода и перехода кривой обучения в стадию стабилизации послеоперационных

Таблица 3. Сравнение непосредственных результатов открытых и минимально инвазивных резекций печени по поводу метастазов колоректального рака

\begin{tabular}{|c|c|c|c|c|}
\hline \multirow[t]{2}{*}{ Параметр } & \multirow[t]{2}{*}{ Все пациенты } & \multicolumn{2}{|c|}{ Группа исследования } & \multirow[t]{2}{*}{$p$} \\
\hline & & $\begin{array}{c}\text { минимально } \\
\text { инвазивные } \\
\text { резекции }\end{array}$ & $\begin{array}{l}\text { открытые } \\
\text { резекции }\end{array}$ & \\
\hline Отступ, мм, M (min - max) & $6(1-20)$ & $6(1-20)$ & $6(1-20)$ & 0,996 \\
\hline Частота применения приема Прингла, n (\%) & $40(48)$ & $29(57)$ & $11(34)$ & 0,228 \\
\hline Длительность операции, мин, M (min - max) & $365(85-780)$ & $360(85-755)$ & $372(180-780)$ & 0,680 \\
\hline Объем кровопотери, мл, M (min - max) & $414(0-3300)$ & $308(0-3300)$ & $583(50-3000)$ & 0,012 \\
\hline Частота переливания эритроцитарной массы, n (\%) & $8(10)$ & $3(6)$ & $5(16)$ & 0,188 \\
\hline $\begin{array}{l}\text { Частота переливания свежезамороженной плазмы, } \\
\text { n (\%) }\end{array}$ & $12(15)$ & $5(10)$ & $7(22)$ & 0,193 \\
\hline Длительность нахождения в ОРИТ, сут, М (min - max) & $2(0-13)$ & $2(0-13)$ & $2(0-13)$ & 0,607 \\
\hline Частота тяжелых осложнений, n (\%) & $18(22)$ & $10(20)$ & $8(25)$ & 0,643 \\
\hline $\begin{array}{l}\text { Длительность послеоперационного стационарного } \\
\text { лечения, сут, М (min - max) }\end{array}$ & $10(4-29)$ & $9(4-29)$ & $12(5-26)$ & $<0,001$ \\
\hline
\end{tabular}


Таблица 4. Распределение осложнений по степени тяжести (Clavien - Dindo), n (\%)

\begin{tabular}{llll}
\hline Степень тяжести & \multicolumn{2}{c}{ Вариант доступа } & \multicolumn{2}{c}{ Всего } \\
\cline { 2 - 3 } & минимально инвазивный & открытый & $4(5)$ \\
\hline II & $2(4)$ & $2(6)$ & $3(4)$ \\
IIla & $2(4)$ & $1(3)$ & $15(18)$ \\
IIIb & $8(16)$ & $7(22)$ & $1(1)$ \\
IV & $1(2)$ & 0 & 0 \\
V & 0 & 0 & $2(3)$ \\
Итого & $1(2)$ & $1(3)$ & $25(31)$ \\
\hline
\end{tabular}

Таблица 5. Характер осложнений, потребовавших инструментальных вмешательств под местной или общей анестезией (более II степени по Clavien - Dindo), n (\%)

\begin{tabular}{|c|c|c|c|}
\hline \multirow[t]{2}{*}{ Осложнение } & \multicolumn{2}{|c|}{ Вариант доступа } & \multirow[t]{2}{*}{ Всего } \\
\hline & $\begin{array}{l}\text { минимально } \\
\text { инвазивный }\end{array}$ & открытый & \\
\hline Жидкостное скопление (серозно-геморрагическое) & $2(4)$ & $5(16)$ & $7(9)$ \\
\hline Билома & $5(10)$ & 0 & $5(6)$ \\
\hline Гематома & 0 & $1(3)$ & $1(1)$ \\
\hline Гидроторакс & $1(2)$ & 0 & $1(1)$ \\
\hline Пневмоторакс & $1(2)$ & 0 & $1(1)$ \\
\hline Нагноение раны & 0 & $1(3)$ & $1(1)$ \\
\hline Острая печеночная недостаточность & $1(2)$ & $1(3)$ & $2(2)$ \\
\hline Итого & $10(20)$ & $8(25)$ & $18(21)$ \\
\hline
\end{tabular}

показателей, изучено меньше. Тем не менее, согласно тем же авторам, длительность второго периода также включает не менее 50-70 резекций печени. Таким образом, общая длительность кривой обучения, необходимая для достижения приемлемых результатов, в том числе после выполнения сложных резекций печени, составляет порядка 100-120 резекций. В представленном в настоящей публикации опыте общее число минимально инвазивных резекций печени превысило 200 вмешательств. В связи с этим представилась возможность проведения сравнения непосредственных результатов минимально инвазивных резекций и открытых операций аналогичного объема.
Для оценки сопоставимости групп проведено сравнение исходных данных минимально инвазивных и открытых резекций. Для этого прежде всего изучены показатели, характеризующие техническую сложность выполненных резекций печени. С этой целью использован так называемый индекс сложности, представляющий собой интегральный показатель, учитывающий размер, топографию опухоли, взаимоотношения с крупными сосудами и другие факторы, влияющие на риск выполнения минимально инвазивной резекции печени. ИСЛРП, отражающий степень риска операции у конкретного пациента, предложен D. Ban и соавт. [5]. Помимо этого проведено сравнение групп по каждому из составляющих 
индекс параметров, а также по тем факторам, которые не учитывает ИСЛРП, но которые влияют на исход резекции печени (число очагов и билобарный характер поражения).

Необходимость сравнения лапароскопических и открытых резекций печени продиктована единичными публикациями по этой проблеме в отечественной литературе, а также наличием единственного зарубежного одноцентрового рандомизированного проспективного исследования, показавшего отсутствие разницы в непосредственных результатах открытых и лапароскопических резекций печени у пациентов, оперированных по поводу метастазов колоректального рака $[2,8]$. В результате анализа опубликованной литературы три последовательно принятых консенсуса по лапароскопическим резекциям печени - в 2008, 2014 и 2017 гг. - не нашли достаточно аргументов для рекомендации применения лапароскопических резекций в широкой практике, в том числе в специализированных отделениях хирургической гепатологии, за исключением операций низкой сложности (резекции передних и латеральных сегментов). Обширные резекции и резекции труднодоступных сегментов остаются уделом специалистов, обладающих достаточным опытом резекционной и лапароскопической хирургии [9-11]. Многие из рекомендаций последнего консенсуса получили низкий уровень доказательности.

Проведенное нами ретроспективное сравнение показало, что минимально инвазивные резекции сопровождались меньшей кровопотерей и меньшей продолжительностью послеоперационного стационарного лечения. Частота тяжелых осложнений (более II степени по Clavien Dindo) в группах не различалась. Ближайшие онкологические результаты (ширина отступа края резекции от поверхности опухоли) также не различались. Правомочность сравнения приведенных групп минимально инвазивных и открытых резекций обоснована сопоставимостью исходных предоперационных показателей, отражающих риски предстоящей операции (коэффициент сложности), а также степени распространенности метастатического поражения печени (статистически значимых различий по этим показателям не выявлено). Совокупный опыт минимально инвазивных резекций печени, накопленный в нашем центре, представляется достаточным для корректного сравнения с технологией открытых резекций с учетом необходимости преодоления кривой обучения новой хирургической технологии.
Несмотря на то что с момента выполнения первой операции прошло уже четверть века, концепция лапароскопической резекции печени окончательно не сформирована. Существуют различные интерпретации технического исполнения резекций одинакового объема, нет убедительных данных, подтверждающих одинаковую безопасность и эффективность минимально инвазивного и открытого доступа при выполнении резекций повышенной сложности. Многие специализированные центры продолжают накапливать опыт реализации вариантов исполнения лапароскопической резекции печени, которые могут меняться в зависимости от локализации, размера и числа очаговых образований, контакта с крупными сосудами и качества паренхимы печени. По мере накопления опыта изменяются представления о возможности и целесообразности выполнения резекций печени разного объема. Соответственно, меняются и результаты операций. В связи с этим на повестке дня еще много вопросов, которые мало освещены в литературе и остались за рамками настоящей публикации.

К недостаткам нашего исследования отнесем его ретроспективный характер, а также отсутствие применения статистических методов, направленных на дополнительное выравнивание групп по показателям, которые помимо изучаемого фактора могут влиять на результат сравнения. Такие противоречия минимизируются в проспективных рандомизированных исследованиях, в меньшей мере - при выполнении псевдорандомизации ретроспективного материала (propensity score matching).

\section{Заключение}

На основании проведенного исследования можно предположить, что при условии накопления опыта минимально инвазивных резекций печени в условиях специализированного центра хирургической гепатологии, располагающего опытом регулярных лапароскопических вмешательств, уровень технического исполнения операции может быть повышен до сопоставимого с открытыми резекциями высокой сложности без изменения частоты и характера осложнений, но с улучшением непосредственных результатов в виде сокращения сроков лечения пациентов в стационаре. Дальнейшее накопление опыта и проведение более узконаправленных исследований позволят внести дополнительную ясность в вопросы безопасности и надежности минимально инвазивных резекций печени. (е)

\footnotetext{
Конфликт интересов Авторы декларируют отсутствие явных и потенциальных конфликтов интересов, связанных с публикацией настоящей статьи.

Финансирование Работа проведена без привлечения дополнительного финансирования со стороны третьих лиц.
} 


\section{Литература}

1. Gagner M, Rheault M, Dubuc J. Laparoscopic partial hepatectomy for liver tumor (abstract). Surg Endosc. 1992;6:99.

2. Fretland ÅA, Dagenborg VJ, Bjørnelv GMW, Kazaryan AM, Kristiansen R, Fagerland MW, Hausken J, Tønnessen Tl, Abildgaard A, Barkhatov L, Yaqub S, Røsok BI, Bjørnbeth BA, Andersen MH, Flatmark K, Aas E, Edwin B. Laparoscopic versus open resection for colorectal liver metastases: The OSLO-COMET randomized controlled trial. Ann Surg. 2018;267(2):199-207. doi: 10.1097/ SLA.0000000000002353.

3. Wong-Lun-Hing EM, van Dam RM, van Breukelen GJ, Tanis PJ, Ratti F, van Hillegersberg R, Slooter GD, de Wilt JH, Liem MS, de Boer MT, Klaase JM, Neumann UP, Aldrighetti LA, Dejong $\mathrm{CH}$; ORANGE II Collaborative Group. Randomized clinical trial of open versus laparoscopic left lateral hepatic sectionectomy within an enhanced recovery after surgery programme (ORANGE II study). Br J Surg. 2017;104(5):525-35. doi: 10.1002/bjs.10438.

4.Xie SM, Xiong JJ, Liu XT, Chen HY, Iglesia-García D, Altaf K, Bharucha S, Huang W, Nunes QM, Szatmary P, Liu XB. Laparoscopic Versus Open Liver Resection for Colorectal Liver Metastases: A Comprehensive Systematic Review and Meta-analysis. Sci Rep. 2017;7(1): 1012. doi: 10.1038/s41598-017-00978-z.

5. Ban D, Tanabe M, Ito H, Otsuka $Y$, Nitta H, Abe $Y$, Hasegawa $Y$, Katagiri T, Takagi $C$, Itano O, Kaneko H, Wakabayashi G. A novel difficulty scoring system for laparoscopic

\section{References}

1. Gagner M, Rheault M, Dubuc J. Laparoscopic partial hepatectomy for liver tumor (abstract). Surg Endosc. 1992;6:99.

2. Fretland ÅA, Dagenborg VJ, Bjørnelv GMW, Kazaryan AM, Kristiansen R, Fagerland MW, Hausken J, Tønnessen Tl, Abildgaard A, Barkhatov L, Yaqub S, Røsok BI, Bjørnbeth $\mathrm{BA}$, Andersen MH, Flatmark K, Aas E, Edwin B. Laparoscopic versus open resection for colorectal liver metastases: The OSLO-COMET randomized controlled trial. Ann Surg. 2018;267(2):199-207. doi: 10.1097/ SLA.0000000000002353.

3. Wong-Lun-Hing EM, van Dam RM, van Breukelen GJ, Tanis PJ, Ratti F, van Hillegersberg R, Slooter GD, de Wilt JH, Liem MS, de Boer MT, Klaase JM, Neumann UP, Aldrighetti LA, Dejong $\mathrm{CH}$; ORANGE II Collaborative Group. Randomized clinical trial of open versus laparoscopic left lateral hepatic sectionectomy within an enhanced recovery after surgery programme (ORANGE II study). Br J Surg. 2017;104(5):525-35. doi: 10.1002/ bjs.10438. liver resection. J Hepatobiliary Pancreat Sci. 2014;21(10):745-53. doi: 10.1002/jhbp.166.

6. Tomassini F, Scuderi V, Colman R, Vivarelli M, Montalti R, Troisi RI. The single surgeon learning curve of laparoscopic liver resection: A continuous evolving process through stepwise difficulties. Medicine (Baltimore). 2016;95(43):e5138. doi: 10.1097/ MD.0000000000005138.

7. Kluger MD, Vigano L, Barroso R, Cherqui D. The learning curve in laparoscopic major liver resection. J Hepatobiliary Pancreat Sci. 2013;20(2): 131-6. doi: 10.1007/s00534-012-0571-1.

8. Ефанов МГ, Алиханов РБ, Цвиркун ВВ, Казаков ИВ, Ким ПП, Ванькович АН, Ахаладзе ДГ, Грендаль КД, Заманов ЭН. Ближайшие и отдаленные результаты лапароскопических и робот-ассистированных резекций печени. Оценка опыта специализированного центра. Анналы хирургической гепатологии. 2018;23(1):38-46. doi: 10.16931/19955464.2018-1-38-46.

9. Buell JF, Cherqui D, Geller DA, O'Rourke N, Iannitti D, Dagher I, Koffron AJ, Thomas M, Gayet B, Han HS, Wakabayashi G, Belli G, Kaneko H, Ker CG, Scatton O, Laurent A, Abdalla EK, Chaudhury P, Dutson E, Gamblin C, D'Angelica M, Nagorney D, Testa G, Labow D, Manas D, Poon RT, Nelson H, Martin R, Clary B, Pinson WC, Martinie J, Vauthey JN, Goldstein R, Roayaie S, Barlet D, Espat J, Abecassis M, Rees $M$, Fong $Y$, McMasters KM, Broelsch $C$, Busuttil R, Belghiti J, Strasberg S, Chari RS; World Consensus Conference on Laparoscop-

4. Xie SM, Xiong JJ, Liu XT, Chen HY, Iglesia-García D, Altaf K, Bharucha S, Huang W, Nunes QM, Szatmary P, Liu XB. Laparoscopic Versus Open Liver Resection for Colorectal Liver Metastases: A Comprehensive Systematic Review and Meta-analysis. Sci Rep. 2017;7(1): 1012. doi: 10.1038/s41598-017-00978-z.

5. Ban D, Tanabe $M$, Ito $H$, Otsuka $Y$, Nitta $H$, Abe $Y$, Hasegawa $Y$, Katagiri T, Takagi $C$, Itano O, Kaneko H, Wakabayashi G. A novel difficulty scoring system for laparoscopic liver resection. J Hepatobiliary Pancreat Sci. 2014;21(10):745-53. doi: 10.1002/jhbp. 166.

6. Tomassini F, Scuderi V, Colman R, Vivarelli M, Montalti R, Troisi RI. The single surgeon learning curve of laparoscopic liver resection: A continuous evolving process through stepwise difficulties. Medicine (Baltimore). 2016;95(43):e5138. doi: 10.1097/ MD. 0000000000005138 .

7. Kluger MD, Vigano L, Barroso R, Cherqui D. The learning curve in laparoscopic major liver resection. J Hepatobiliary Pancreat Sci. 2013;20(2): 131-6. doi: 10.1007/s00534-012-0571-1. ic Surgery. The international position on laparoscopic liver surgery: The Louisville Statement, 2008. Ann Surg. 2009;250(5):825-30. doi: 10.1097/SLA.0b013e3181b3b2d8.

10. Wakabayashi G, Cherqui D, Geller DA, Buell JF, Kaneko $\mathrm{H}$, Han HS, Asbun $\mathrm{H}$, O'Rourke $\mathrm{N}$, Tanabe M, Koffron AJ, Tsung A, Soubrane O, Machado MA, Gayet B, Troisi RI, Pessaux P, Van Dam RM, Scatton O, Abu Hilal M, Belli G, Kwon $\mathrm{CH}$, Edwin $\mathrm{B}$, Choi GH, Aldrighetti LA, Cai X, Cleary S, Chen KH, Schön MR, Sugioka A, Tang CN, Herman P, Pekolj J, Chen XP, Dagher I, Jarnagin W, Yamamoto M, Strong R, Jagannath $\mathrm{P}$, Lo CM, Clavien PA, Kokudo N, Barkun J, Strasberg SM. Recommendations for laparoscopic liver resection: a report from the second international consensus conference held in Morioka. Ann Surg. 2015;261(4):619-29. doi: 10.1097/SLA.0000000000001184.

11. Abu Hilal M, Aldrighetti $L$, Dagher I, Edwin B, Troisi Rl, Alikhanov R, Aroori S, Belli G, Besselink $M$, Briceno J, Gayet B, D'Hondt $M$, Lesurtel $M$, Menon K, Lodge $P$, Rotellar F, Santoyo J, Scatton O, Soubrane O, Sutcliffe R, Van Dam R, White S, Halls MC, Cipriani F, Van der Poel M, Ciria R, Barkhatov L, Gomez-Luque $Y$, Ocana-Garcia S, Cook A, Buell J, Clavien PA, Dervenis C, Fusai G, Geller D, Lang H, Primrose J, Taylor M, Van Gulik T, Wakabayashi G, Asbun $\mathrm{H}$, Cherqui D. The Southampton Consensus Guidelines for laparoscopic liver surgery: from indication to implementation. Ann Surg. 2018;268(1):11-8. doi: 10.1097/ SLA.0000000000002524.

8. Efanov MG, Alikhanov RB, Tsvirkun VV, Kazakov IV, Kim PP, Vankovich AN, Akhaladze DG, Grendal KD, Zamanov EN. Early and long-term outcomes of laparoscopic and robot-assisted liver resections. Specialized center's experience. Annals of HPB surgery. 2018;23(1):3846. Russian. doi: 10.16931/1995-5464.2018-138-46.

9. Buell JF, Cherqui D, Geller DA, O'Rourke N, Iannitti D, Dagher I, Koffron AJ, Thomas $M$, Gayet B, Han HS, Wakabayashi G, Belli G, Kaneko H, Ker CG, Scatton O, Laurent A, Abdalla EK, Chaudhury P, Dutson E, Gamblin C, D'Angelica M, Nagorney D, Testa G, Labow D, Manas D, Poon RT, Nelson H, Martin R, Clary B, Pinson WC, Martinie J, Vauthey JN, Goldstein R, Roayaie S, Barlet D, Espat J, Abecassis $M$, Rees M, Fong Y, McMasters KM, Broelsch C, Busuttil R, Belghiti J, Strasberg S, Chari RS; World Consensus Conference on Laparoscopic Surgery. The international position on laparoscopic liver surgery: The Louisville Statement, 2008. Ann Surg. 2009;250(5):825-30. doi: 10.1097/SLA.0b013e3181b3b2d8. 
10. Wakabayashi G, Cherqui D, Geller DA, Buell JF, Kaneko $\mathrm{H}$, Han HS, Asbun $\mathrm{H}$, O'Rourke $\mathrm{N}$, Tanabe M, Koffron AJ, Tsung A, Soubrane O, Machado MA, Gayet B, Troisi RI, Pessaux P, Van Dam RM, Scatton O, Abu Hilal M, Belli G, Kwon $\mathrm{CH}$, Edwin B, Choi GH, Aldrighetti LA, Cai X, Cleary S, Chen KH, Schön MR, Sugioka A, Tang CN, Herman P, Pekolj J, Chen XP, Dagher I, Jarnagin W, Yamamoto $M$, Strong $R$, Jagannath P, Lo CM, Clavien PA, Kokudo N,
Barkun J, Strasberg SM. Recommendations for laparoscopic liver resection: a report from the second international consensus conference held in Morioka. Ann Surg. 2015;261(4):619-29. doi: $10.1097 /$ SLA.0000000000001184.

11. Abu Hilal M, Aldrighetti L, Dagher I, Edwin B, Troisi RI, Alikhanov R, Aroori S, Belli G, Besselink M, Briceno J, Gayet B, D'Hondt M, Lesurtel M, Menon K, Lodge P, Rotellar F, Santoyo J, Scatton O, Soubrane O, Sutcliffe R, Van
Dam R, White S, Halls MC, Cipriani F, Van der Poel M, Ciria R, Barkhatov L, Gomez-Luque $Y$, Ocana-Garcia S, Cook A, Buell J, Clavien PA, Dervenis C, Fusai G, Geller D, Lang H, Primrose J, Taylor M, Van Gulik T, Wakabayashi G, Asbun H, Cherqui D. The Southampton Consensus Guidelines for laparoscopic liver surgery: from indication to implementation. Ann Surg. 2018;268(1):11-8. doi: 10.1097/ SLA. 0000000000002524 .

\title{
Short-term results after minimally invasive and open liver resection for liver metastases of colorectal cancer: a single center experience
}

\author{
M.G. Efanov' • R.B. Alikhanov' • V.V. Tsvirkun ${ }^{1}$ • I.V. Kazakov' • \\ P.P. Kim • A.N. Vankovich' • K.D. Grendal² • E.N. Zamanov²
}

Rationale: Until now, safety of minimally invasive liver resection (MILR) has not been studied sufficiently. Aim: To assess immediate results of MILR and open type resections in patients with colorectal metastases, performed in the Russian center of surgical hepatology specialized at implementation of minimally invasive techniques. Materials and methods: This was a retrospective observational case-control study. Patients who underwent surgery for isolated liver metastases of colorectal cancer in a single center from October 2013 to February 2018 were included into the study. Results: As per December 2017, over 500 resections have been performed in the study center, including 226 MILR. One hundred two patients underwent open resection and MILR for colorectal metastases. From 83 patients enrolled into the study, 51 (61\%) had MILR, including 7 robotic MILR. The open resection and MILR groups did not differ in terms of gender, age, ASA score, primary tumors location and stage by the time of primary intervention. There were no between-group differences for factors that determine the tumor spread and influence the resection problems, i.e. the difficulty index of MILR, rate of anatomic resection, resection of complex segments, vascular involvement, size and number of metastases, multiple liver lesions and bilobar metastases. No difference was found for immediate outcomes in terms of frequency of the free surgical margin $>2 \mathrm{~mm}$, rate of the Pringle maneuver implementation, duration of the procedure, blood components transfusion, severe complications (Clavien-Dindo Grade $>$ II), and time in intensive care unit. Compared to open procedures, MILR were associated with significantly less blood loss: $583(50-3000) \mathrm{mL}$ vs. $308(0-3300) \mathrm{mL} \quad(p=0.012)$, respectively, and shorter duration of hospital stay: 10 (4-29) days vs. $9(4-29)$ days $(p<0.001)$, respectively. Conclusion: In a specialized surgical hepatology center, MILR can be performed equally to complex open procedures without changes in the rates and types of complications, but with an improvement of immediate outcomes.

Key words: minimally invasive surgery, laparoscopic liver resection, colorectal metastases

For citation: Efanov MG, Alikhanov RB, Tsvirkun W, Kazakov IV, Kim PP, Vankovich AN, Grendal KD, Zamanov EN. Short-term results after minimally invasive and open liver resection for liver metastases of colorectal cancer: a single center experience. Almanac of Clinical Medicine. 2018;46(6):584-91. doi: 10.18786/2072-0505-2018-46-6-584-591.

Received 9 June 2018; accepted 6 August 2018
Mikhail G. Efanov - MD, PhD, Head of the Department of Hepato-Pancreatobiliary Surgery' $\triangle 86$ Shosse Entuziastov, Moscow, 111123, Russian Federation. Tel.: +7 (916) 1058830

E-mail:m.efanov@mknc.ru

Ruslan B. Alikhanov - MD, PhD, Chief of the Division of Hepato-Pancreatobiliary Surgery ${ }^{1}$

Victor V. Tsvirkun - MD, PhD, Professor, Chief Research Fellow ${ }^{1}$

Ivan V. Kazakov - MD, PhD, Senior Research Fellow Division of Hepato-Pancreatobiliary Surgery'

Pavel P. Kim - Research Fellow, Division of HepatoPancreatobiliary Surgery'

Andrey N. Vankovich - MD, PhD, Research Fellow, Division of Hepato-Pancreatobiliary Surgery'

Konstantin D. Grendal - Postgraduate Student Chair of Faculty Surgery No. $2^{2}$

Ehtibar N. Zamanov - Postgraduate Student, Chair of Faculty Surgery No. $2^{2}$ 